\title{
Review on Feature Extraction and Classification of WBC in Bone Marrow for Disease Diagnosis
}

\author{
Shri N. D. Pergad ${ }^{1}$, Dr. S. T. Hamde ${ }^{2}$ \\ Associate Professor, Electronic and Telecommunication Dept., STB COE, Tuljapur, India ${ }^{1}$ \\ Professor, Instrumentation Engineering Dept., SGGSCOE\&T, Nanded, India ${ }^{2}$
}

\begin{abstract}
In this paper, we make a review on different features and classification methods for white blood cells in bone marrow for disease diagnosis. A new set of features based on the wavelet and Radon transform to bone marrow blood cell differential classification solution. The transform based novel features are the coefficient values of decomposition for horizontal, vertical \& diagonal factors with two levels. the factors where the coefficient values are of six in numbers the first and second level coefficient method to the classifiers by the desired output using a previous information of the number of coefficients as a samples in each class. Artificial neural networks Levenberg-Marguardt (LM) back propagation algorithm for validation is applied in the experiments. The results are used in various disease diagnoses.
\end{abstract}

Keywords: review on features, review on classification methods, wavelet transform.

\section{INTRODUCTION}

Bone marrow blood cell differential classification in bone marrow images is steps to achieve the white blood cell differential counting. The two methods to count white blood cells in bone marrow images are the first is differential counts and the second is total count. The total count means the total number of white blood cells with general classification. While, the differential counts are the counts of different cell classification in bone marrow.

The differential count provides important information in diagnosis of diseases such as cancers or leukaemia to doctors. There are cell counter machines for total counts but for The differential counting an expert is required to achieve manual count. which is to be done by with the help of microscope selecting an area of interest in a bone marrow slide, detect a white blood cell, classify it with using his knowledge, and measure the count of the corresponding cell class. All of these processes manually to do would need a smart expert, and is a very laborious work.

White blood cells in bone marrow are classified, Using research tool 'MATLAB' according to their maturation stages. When a bone marrow cell becomes older, its nuclei shape, size and many other features have change. Bone marrow cells in the myelocytic series can be classified into six classes, i.e., myeloblast, promyelocyte, myelocyte, metamyelocyte,band, and polymorphonuclear (PMN) ordered from the youngest to the oldest

Figure 1 shows samples of Bone marrow cells in this series. Even though there are various automatic systems available for counting white blood cells in peripheral blood [13], there is some automatic system for bone marrow cells.

Figure1. Multi Thresholded Cell samples in the myelocytic series row wise: (a) Myeloblast; (b) Promyelocyte; (c) Myelocyte; (d) Metamyelocyte; (e) Band; and (f) PMN. cells [1,2].

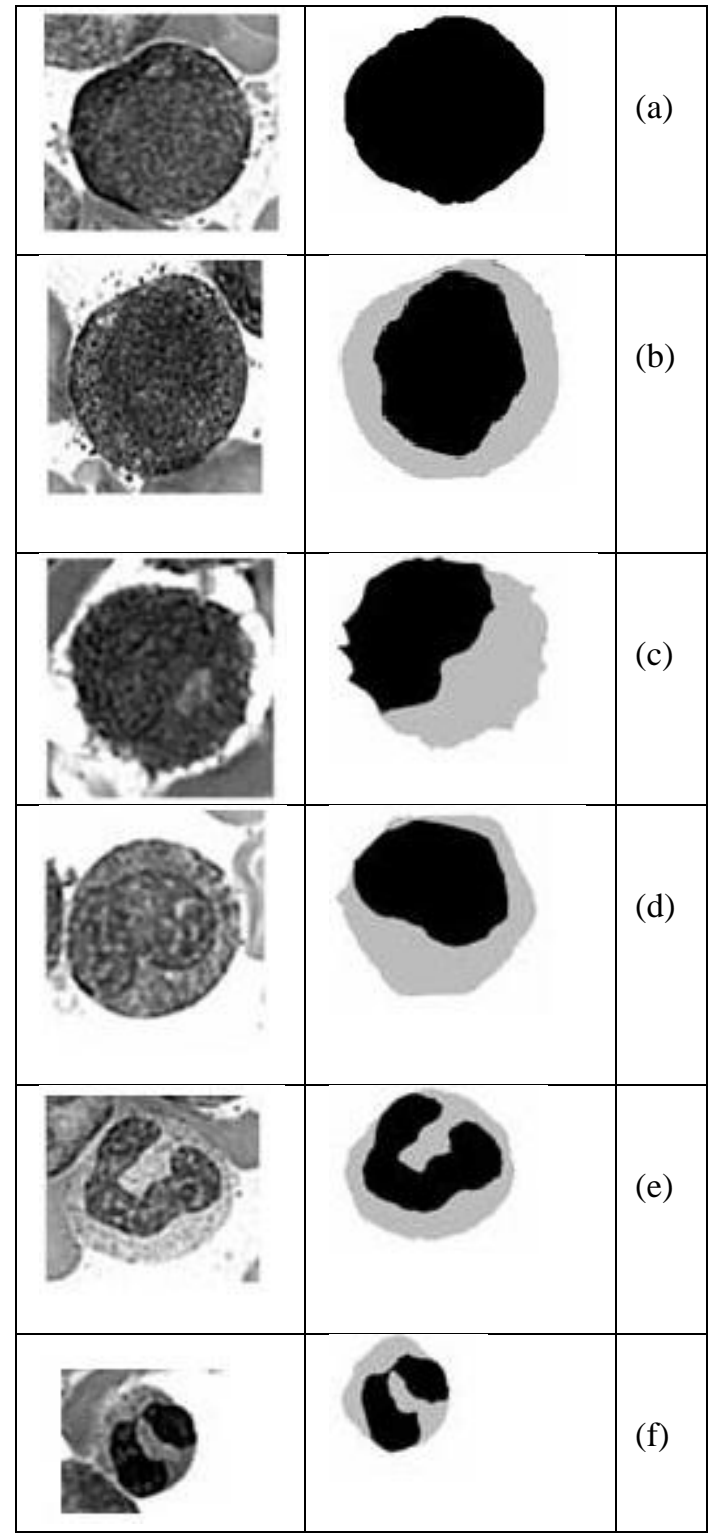


1.1Literature survey:

There are a number of ways in which training data can be applied in classifier methods. A simple classifier is the nearest-neighbour classifier, where each pixel is classified in the same class as the training datum with the closest intensity. The $\mathrm{k}$-nearest-neighbor $(\mathrm{kNN})$ classifier is a generalization of this approach, where the pixel is

classified according to the majority vote of the " $\mathrm{k}$ " closest training data. The $\mathrm{kNN}$ classifier is considered a nonparametric classifier since it makes no underlying assumption about the statistical structure of the data. It is efficient for the classification of the human brain into normal and abnormal[21].

Clustering is an example of unsupervised classification. Classification refers to a procedure that assigns data objects to a set of classes. Unsupervised means that clustering does not depend on predefined classes and training examples while classifying the data objects. Cluster analysis seeks to partition a given data set into groups based on specified features so that the data points within a group are more similar to each other than the points in different groups[22]. Therefore, a cluster is a collection of objects that are similar among themselves and dissimilar to the objects belonging to other clusters. The k-means and fuzzy c-means algorithms start by initializing the cluster centroid. The input vectors (data points) are then allocated (assigned) to one of the existing clusters according to the square of the Euclidean distance from the centroid of clusters, choosing the closest. The mean of each cluster is then computed so as to update the cluster centroid. This update occurs as a result of the change in the membership of each cluster. The processes of re-assigning the input vectors and the update of the cluster centroid is repeated until no more change in the value of any of the cluster centroid. Recently, fuzzy cmeans of unsupervised clustering techniques used on established outstanding results in automated segmenting medical images in a robust manner[23,25,26]. One of the Fuzzy c-means segmentation method benefits, is that it could retain much more information from the original image[22]. Standard classifiers require that the structures to be segmented possess distinct quantifiable features. Because training data can be labeled, classifiers can transfer these labels to new data as long as the feature space sufficiently distinguishes each label as well. Being non-iterative, they are relatively computationally efficient and unlike thresholding methods, they can be applied to multi-channel images. A disadvantage of classifiers is that they generally do not perform any spatial modeling. This weakness has been addressed in recent work extending classifier methods to segmenting images that are corrupted by intensity inhomogeneities[27]. Neighborhood and geometric information have also been incorporated into classifiers[28].

Another disadvantage is the requirement of manual interaction for obtaining training data. Training sets can be acquired for each image that requires segmenting, which is time consuming. However, the use of the same training set for a large number of scans can lead to biased results, which do not take into account anatomical and physiological variability between different subjects[19].
Intelligent classifiers, which have learning capabilities, involve the use of Artificial Intelligence techniques in the classification process, and many, such as Artificial Neural Networks (ANN) have their origins in biological systems. ANNs are typically massively parallel networks of processing elements or nodes that simulate biological systems for learning and decision-making. Each node in an ANN is capable of performing elementary computations. Learning is achieved through the adaptation of weights assigned to the connections between nodes. Further details of the architecture, training and operation of ANNs and a thorough treatment on ANN can be found in the literature[29,30].

The ANN is widely used in medical imaging as a classifier[31,32], where selected input features that have been extracted from the images are input to the ANN, which then determines the weights in a training phase, where the correct classifications are known (supervised training) and the ANN is then used to classify new data. ANNs can also be used in an unsupervised fashion as a clustering method [20,33]. For example to separate between the

blood vessel class and the fat class, three extracted features, narrowness and histogram consistency, were used as the inputs to the ANN[34].

Other medical applications of ANNs include disease diagnosis such as liver cancer detection[35], locating automatically the outline of the lungs in MRI images of the thorax[36], Brain tumour classification[37], and in abnormal retinal image classification[38], An example is lung cancer detection by using ANN and fuzzy C-Mean clustering algorithm [39]. This was a promising field to apply in the classification of WBC differentiated bone marrow cells.

\subsection{Other approaches:}

Model-fitting is a segmentation method that typically fits a simple geometric shape such as an ellipse to the locations of extracted image features in an image[40]. It is a technique which is specialized to the structure being segmented but is easily implemented and can provide good results when the model is appropriate. A more general approach is to fit spline curves or surfaces[41] to the features. The main difficulty with model-fitting is that image features must first be extracted before the fitting can take place. The watershed algorithm uses concepts from mathematical morphology to partition images into homogeneous regions[42]. This method can suffer from over segmentation, which occurs when the image is segmented into an unnecessarily large number of regions. Thus, watershed algorithms in medical imaging are usually followed by a post processing step to merge separate regions that belong to the same structure[43].

Active contour model, also called snakes, is a framework for delineating an object outline from a possibly noisy $2 \mathrm{D}$ image. This framework attempts to minimize an energy associated to the current contour as a sum of an internal and external energy. The external energy is supposed to be minimal when the snake is at the object boundary position. The most straightforward approach consists in giving low values when the regularized gradient around the contour position reaches its peak value. The internal energy is 
supposed to be minimal when the snake has a shape that is supposed to be relevant considering the shape of the sought object. The most straightforward approach grants high energy to elongated contours and to bended/high curvature contours, considering the shape should be as regular and smooth as possible[44].

The SVM (Support vector machine) could be one of the approaches where the classification problem can be restricted to consideration of the two-class problem without loss of generality. In this problem the goal is to separate the two classes by a function which is induced from available examples. The goal is to produce a classifier that will work well on unseen examples, i.e. it generalises well. There are many possible linear classifiers that can separate the data, but there is only one that maximises the margin (maximises the distance between it and the nearest data point of each class). This linear classifier is termed the optimal separating hyperplane[45]. There have been several methods like proposed to solve the problem. Most methods follow the traditional manual count systems, i.e., to detect a cell, extract its features, classify the cell, and then update the count $[3,4]$. Some are based on neural networks with features such as area of cell, area of nuclei, ratio of area of nuclei to cytoplasm, Fourier descriptors of nuclei, some textural features, etc. [5-9]. Some are developed under the mixing theories of the mathematical morphology [11].

In this paper, we will apply artificial neural networks to the white blood cell classification for single cell images for the perfect cell segmentation is available. We propose new features of a bone marrow cell based on wavelet transform coefficients. This paper is organized as follows. Section2 introduces the methodology with wavelet transform theory, neural networks, In section3The extraction of proposed features for classification. also the data base is described. In section 4 the description of computations of results. In section 5 this paper concludes.

\section{METHODOLOGY}

In our research, Artificial neural networks are used as our classifiers in the six class solution. The cell features are mainly extracted from segmented bone marrow cell images with its nucleus and cytoplasm background. Here, we extract six coefficients of Radon and Wavelet transforms as the six features of the cells.

\subsection{Radon and Wavelet Transform Theory:}

Radon Transform forming a very important mathematical tool used in tomography is based upon works of Johann Radon born in 1887 Litom e rice. His doctoral dissertation has been defended in Vienna in 1910 and his most appreciated works were devoted to integral geometry. The Radon Transform[15] belonging to this category introduced in 1917 is defined as a collection of 1D projections around an object at angle intervals . The Radon Transform of a two-dimensional (2-D) function $\mathrm{f}(\mathrm{x}, \mathrm{y})$ is defined as

$$
R(r, \Theta)[f(x, y)]=\int_{+\infty}^{-\infty} \int_{+\infty}^{-\infty} f(x, y) \delta(r, x \cos \Theta-y \sin \Theta) d x d y
$$

where $r$ is the perpendicular distance of a line from the origin and $\Theta$ is the angle formed by the distance vector. A discrete Radon transform called Hough transform has been introduced in 1972 by R. Duda and P. Hart as a tool for image features extraction. Wavelet decomposition using Discrete Wavelet Transform (DWT)[15] provides an image analysis resulting in image decomposition into twodimensional functions of time and scale. The main benefit of DWT is in its multi-resolution time-scale analysis ability. Wavelet functions used for image analysis are derived from the initial function $\mathrm{W}(\mathrm{t})$ forming basis for the set of Functions

$$
\mathrm{Wm}, \mathrm{k}(\mathrm{t})=1 / \sqrt{\mathrm{a}} \mathrm{W} \quad(1 / \mathrm{a}(\mathrm{t}-\mathrm{b}))
$$

Figure 2

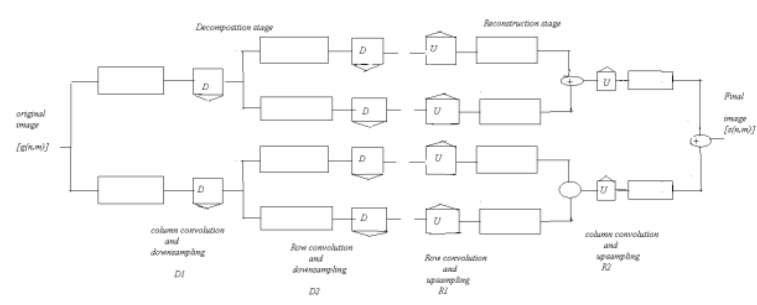

Wavelet transform use in image decomposition and the effect of Haar and Bio3.7 wavelet function dilation to its spectrum compression analysis. The principle of image decomposition and reconstruction for resolution enhancement is presented in Fig. 3. The decomposition stage includes the processing of the image matrix by columns at first using wavelet (high-pass) and scaling (low-pass) function followed by row down sampling by factor D in stage D1. To study this problem let us denote a selected column of the image matrix $[g(n, m)] N, M$ as in image.

$$
\{\mathrm{x}(\mathrm{n})\}_{n=0}^{N-1}=[\mathrm{x}(0), \mathrm{x}(1), \ldots, \mathrm{x}(\mathrm{N}-1)] \mathrm{T} \ldots \ldots .(2 \mathrm{~b})
$$

This image can be analyzed by a half-band low-pass filter with its impulse response

$$
\{\mathrm{s}(\mathrm{n})\}_{n=0}^{L-1}=[\mathrm{s}(0), \mathrm{s}(1), \ldots \cdot \mathrm{s}(\mathrm{L}-1)]
$$

and complementary high-pass filter having impulse response

$$
\{\mathrm{w}(\mathrm{n})\}_{n=0}^{L-1}=[\mathrm{w}(0), \mathrm{w}(1), . \cdot \mathrm{w}(\mathrm{L}-1)] \quad-----(4)
$$

The first stage assumes the convolution of a given image and the appropriate filter for decomposition at first by relations

$$
\mathrm{xl}(\mathrm{n})=\sum_{k=0}^{L-1} \mathrm{~s}(\mathrm{k}) \mathrm{x}(\mathrm{n}-\mathrm{k}) \mathrm{xh}(\mathrm{n})=\sum_{k=0}^{L-1} \mathrm{w}(\mathrm{k}) \mathrm{x}(\mathrm{n}-\mathrm{k})
$$

for all values of $\mathrm{n}$ followed by sub sampling by factor $\mathrm{D}$. In the following decomposition stage D2 the same process is applied to rows of the image matrix followed by row down sampling. The decomposition stage results in this way in four image representing all combinations of lowpass and high-pass initial image matrix processing. The 
reconstruction stage includes row up sampling by factor $U$ be measured, such as the mean, variance, entropy, energy, at first and row convolution in stage $\mathrm{R} 1$. The contrast, and correlation.

corresponding images are then summed. The final step R2 assumes column up sampling and convolution with reconstruction filters followed by summation of the results again.

\subsection{Proposed Method:}

In this section, the rotation-invariant texture-analysis technique using Radon and wavelet transforms is introduced. This technique is depicted in Fig. 3.

Figure 3: Block diagram of the proposed technique

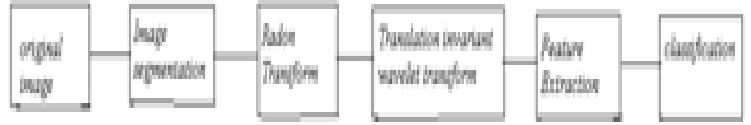

The illustration shows the procedure of proposed method in block diagram. At first we identify all image components using distance and Hough transform. Then we obtain the Radon transform of the image segments and then use a translation-invariant wavelet transform to calculate the frequency components and extract the corresponding features. Rotation of the input image corresponds to the translation of the Radon transform along Fig. 3 shows how the Radon transform changes as the simulated image rotates. The figure presents rotation of the simulated image, whose whole the image components are same, corresponds to a circular shift along. Therefore, using a translation-invariant wavelet transform along, we can produce rotation-invariant features. Fig.4

Figure 4: DWT applied to Radon transform by angle increase step 2 degree .

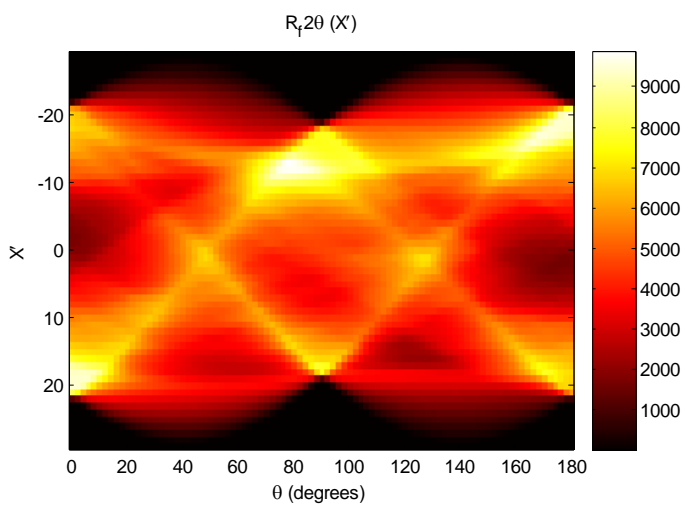

\section{FEATURE EXTRACTIONS}

The various approaches and techniques that have been used to derive texture features from an image[88] are listed below:

(i) Co-occurrence matrices, also called Grey Level Cooccurrence Matrices (GLCM) attempt to capture texture using a sparse representation. Each matrix in the set corresponds to an offset (e.g. 2 pixels down and 1 pixel to the left). The entry in row $i$ and column $j$ of each matrix is the number of pixels in the image of grey level $i$ that have a neighbour of grey level $\mathrm{j}$ in the direction of the offset. From these matrices a number of statistical descriptors can
(ii)The correlation coefficients gives an indication of the similarity between two datasets of different images, while auto-correlation can be used as a measure of the coarseness of texture in different directions, as one of the image datasets is displaced relative to itself.

(iii) The Discrete Fourier Transform and the Discrete Cosine Transform components reflect the spatial structure in an image, and so the significant components can be useful descriptors for classification.

(iv) Grey-scale histogram features provide a concise and useful representation of the intensity levels in a grey-scale image. The histogram-based descriptors include the mean, variance (or its square root, the standard deviation), skewness, energy (used as a measure of uniformity).

(v) Fractals are sometimes used for texture analysis and segmentation because, like texture, they have inherent scales attached to them. Rather than guessing at a scale at which the analysis should proceed, a fractal analysis can yield the fractal dimension of a texture, which should indicate the scale[88].

The main goal of this paper is to show, how is the features are changing by use different transforms by image rotation. Our wish is to have the same image features independently of image rotation. Fig. 5 presents, how passes the features analysis of simulated image, which rotates by angle from $0 \circ$ to $180^{\circ}$ with step $2^{\circ}$. Characteristic image features, shown in Table. 1 are computed of the diagonal DWT transform coefficients in the first and the second decomposition levels rotated image by angle $\Theta=2 \circ$ and they are shown in figure as a colored dots. The dots in the figure, presenting features, are evaluated by the DWT applied to the Radon transform(RT) of rotated image
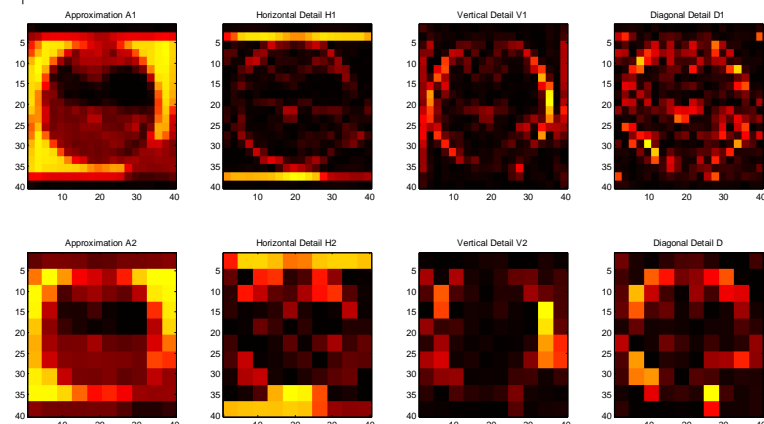

Figure 5: Figure presents the rotation of simulated image, DWT applied to Radon transform, decomposition of the Radon transform image into 1 \& 2 level, Below comparison of image features evaluated by methods mentioned in text before Table1:Comparison of features evaluated for simulated image presenting column F1 as a diagonal DWT coefficients in the first decomposition level and column F2 as a coeffs. in the second decomposition. level, for 3 different techniques (i) HWT, (ii) BOWT and (iii) RHW

These features are for single cell(myeloblast),similarly other remaining are extracted. There are 720 wbc cells which features are extracted. 
INTERNATIONAL JOURNAL OF INNOVATIVE RESEARCH IN ELECTRICAL, ELECTRONICS, INSTRUMENTATION AND CONTROL ENGINEERING Vol. 4, Issue 1, January 2016

Table1:Features of Simulated Image

\begin{tabular}{|c|c|c|c|c|c|c|}
\hline \multirow{2}{*}{$\begin{array}{l}\text { An } \\
\text { gle }\end{array}$} & \multicolumn{2}{|c|}{ HWT } & \multicolumn{2}{|c|}{ BOWT } & \multicolumn{2}{|c|}{ RHWT } \\
\hline & F1 & F2 & F1 & F2 & F1 & F2 \\
\hline 0 & 3.750 & $\begin{array}{l}11.18 \\
7\end{array}$ & 0.187 & $\begin{array}{l}11.34 \\
3\end{array}$ & 4.015 & 13.50 \\
\hline 2 & 3.750 & $\begin{array}{l}11.18 \\
7\end{array}$ & 0.672 & 9.044 & 4.015 & 13.50 \\
\hline 4 & 0.750 & $\begin{array}{l}11.18 \\
7\end{array}$ & 0.507 & $\begin{array}{l}12.75 \\
1\end{array}$ & 1.629 & 13.50 \\
\hline 6 & 0.750 & $\begin{array}{l}11.18 \\
7\end{array}$ & 0.380 & 0.220 & 1.629 & 13.50 \\
\hline 8 & 1.500 & 1.250 & 0.612 & 5.213 & 1.935 & 5.177 \\
\hline 10 & 1.500 & 1.250 & 0.293 & 2.224 & 1.935 & 5.177 \\
\hline 12 & 0.750 & 1.250 & 0.215 & 0.517 & 0.233 & 5.177 \\
\hline 14 & 0.750 & 1.250 & 0.976 & 3.014 & 0.233 & 5.177 \\
\hline 16 & 2.750 & 0.500 & 1.246 & 2.643 & 0.654 & 8.063 \\
\hline
\end{tabular}

\section{CONCLUSION}

We demonstrate that features based on the Radon and wavelet transform coefficients with two decomposition levels .the pair coefficients are selected from each transform having six features for each angle step up to $18^{0}$ rotation, these features are normalized with maximum value of coefficients. the maximum value of a each level is to be used for normalization. these values are useful in the automatic white blood cell classification. The detail information about ANN algorithm like performance characteristics, training plots, and receiver operating characteristics are discussed in next paper. We propose the differential count with this classification. This computation increases the classification rate on the test sets which we can claim that it increase the generalization of the classifier. The features we use in the computations heavily rely on the hand-segmented images. The future work is to incorporate the automatic differential cell classification to the system. Which is used in the various disease diagnosis. This is to be applied with disease analysis data and will be make the conclusions.

\section{ACKNOWLEDGMENT}

The author thanks Dr. V.M. Manure, DR.VMM's, Govt. Medical College, Solapur, M.S. INDIA. For providing the data and the fundamental pathology information. Finally, I acknowledge my guide and co-author for the contribution to Dr. S.T. Hamde, SGGSCE\&T, Nanded. through many technical discussions and to supporting this work.

\section{REFERENCES}

[1] Diggs, L. W., Sturm D., and Bell A., The Morphology of Human Blood Cells, Abbott Laboratories, Abbott Park, 1985.

[2] V. Minnich, Immature Cells in the Granulocytic,Monocytic, and Lymphocytic Series, American Society of Clinical Pathologists Press, Chicago, 1982.

[3] S. S. S. Poon, R. K. Ward, and B. Palcic, "Automated Image Detection and Segmentation in Blood Smears," Cytometry, vol. 13, pp. 766-774, 1992.

[4] S. Sohn, Bone Marrow White Blood Cell Classification, Master's Project, University of Missouri-Columbia, 1999.

[5] N. Theera-Umpon, Morphological Granulometric Estimation with Random Primitives and Applications to Blood Cell Counting, Doctoral Dissertation, University of Missouri,2000.

[6] N. Theera-Umpon, and P. D. Gader, "Counting White Blood Cells
Using Morphological Granulometries," Journal of Electronic Imaging, vol. 9, no. 2, pp. 170-177, 2000.

[7] N. Theera-Umpon, E. R. Dougherty, and P. D. Gader, "NonHomothetic Granulometric Mixing Theory with Application to Blood Cell Counting," Pattern Recognition, vol. 34, no. 12, pp. 2547- 2560, 2001.

[8] N. Theera-Umpon and P. D. Gader, "Training Neural Networks to Count White Blood Cells via a Minimum Counting Error Objective Function," 15th Intl. Conf. on Pattern Recognition, pp. 299- 302, Barcelona, Spain, September 2000.

[9] N. Theera-Umpon, and P. D. Gader, "System Level Training of Neural Networks for Counting White Blood Cells", IEEE Trans. Systems, Man, and Cybernetics Part C: Applications and Reviews, Vol. 32, No. 1, pp. 48-53, 2002.

[10] G. Matheron, Random Sets and Integral Geometry, Wiley, New York, 1975.

[11] J. Serra, Image Analysis and Mathematical Morphology, Academic Press, New York, 1983.

[12] Hematopietic System I: Peripharal Blood Reading: Gartner and Hiatt, pages 89-101

[13] Acute Myeloid Leukemia, from: Journal 'Blood'

[14] E. R. Dougherty, Random Processes for Image and Signal Processing, SPIE Press, Bellingham, Washington, and IEEE Press, New York, 1999.

[15] Radon and Wavelet Transform Theory, from: IMPT and ITT.

[16] S. Haykin, Neural Networks: A Comprehensive Foundation, 2nd Ed., Prentice-Hall, Upper Saddle River, NJ, 1999.

[17] H. Demuth and M. Beale, Neural Network Toolbox: For Use with MATLAB, Mathworks, Natick, 1998.

[18] Dr. S.T. Hamde ${ }^{(2)}$ Mr. N.D.Pergad ${ }^{(1)}$ 'Wavelet and Radon transform in feature extraction of blood cell images',International conference proccedings, at Kanyakumari,Dec.2012

[19] D. L. Phaméê, C. Xué, J. L. Prince, "A Survey of Current Methods in Medical Image", Annual Review of Biomedical Engineering, 1998.

[20] J. C. Bezdek, L. O. Hall, and L. P. Clarke. "Review of MR image segmentation techniques using pattern recognition". Med. Phys., vol. 20, pp. 1033-1048, 1993.

[21] N. Hema Rajini, R. Bhavani, "Classification of MRI Brain Images using k- Nearest Neighbor and Artificial Neural Network", IEEEInternational Conference on Recent Trends in Information Technology, 2011.

[22] N. H. Rajini, R. Bhavani, "Enhancing K-means and Kernelized Fuzzy C-means Clustering with Cluster Center Initialization in Segmenting MRI brain images". 2011. Available at: $\mathrm{http} / / /$ ieeexplore.ieee.org/xpl/articleDetails.jsp?reload=true\&arnum ber $=5941697$.

[23] S. Deelers, S. Auwatanamongkol, "Enhancing K-Means Algorithm with Initial Cluster Centers Derived from Data Partitioning along the Data Axis with the Highest Variance," International Journal of Computer Science, vol. 2, p. 247, 2007.

[24] A. M. Fahim, A. M. Salem, F. A. Torkey, M. A. Ramadan, "An Efficient enhanced k-means clustering algorithm", Journal of Zhejiang University, vol. 10: 1626-1633, 2006.

[25] K. Arai and A. R. Barakbah, "Hierarchical K-means: an algorithm for Centroids initialization for k-means," Saga University, vol. 36, pp. 25-31, 2007.

[26] R. J. Hathaway, J. C. Bezdek, "Extending fuzzy and probabilistic clustering to very large datasets", Computational Statistical and Data Analysis vol. 51, pp. 215-234, 2006.

[27] W. M. Wells, W. E. L. Grimson, R. Kikins, and F. A. Jolesz, "Adaptive segmentation of MRI data", IEEE Trans. on Medical imaging, vol. 15, pp. 429-442, 1996

[28] T. Kapur, W. E. L. Grimson, R. Kikinis, and W.M. Wells, "Enhanced spatial priors for segmentation of magnetic resonance imagery". In Proc. 1st Int. Conf. Med. Im. Comput. Comp. Assist. Interven, pp. 457-468, 1998. [29] J. W. Clark, "Neural network modeling", Phys. Med. Biol., vol. 36, pp. 1259-1317, 1991.

[30] S. Haykin. Neural networks: a comprehensive foundation. New York: Macmillan College Publishing Company, 1994.

[31] L. O. Hall, A. M. Bensaid, L. P. Clarke, R. P. Velthuizen, M. S. Silbiger, and J. C. Bezdek," A comparison of neural network and fuzzy clustering techniques in segmenting magnetic resonance images of the brain", IEEE Trans. Neural Networks, vol. 3: pp. 672$682,1992$.

[32] E. Gelenbe, Y. Feng, and K. R. R. Krishnan, "Neural network methods for volumetric magnetic resonance imaging of the human 
brain", Proc. IEEE, vol. 84: pp. 1488-1496, 1996.

[33] W. E. Reddick, J. O. Glass, E. N. Cook, T. D. Elkin, and R. J. Deaton. "Automated segmentation and classification of multispectral magnetic resonance images of brain using artificail neural networks", IEEE Trans. On Medical imaging, vol. 16, pp. 911-918, 1997. 182

[34] Meta data offer new knowledge, Analytics: Supervised vs. Unsupervised learning. Available at: http://monkpublic.library.illinois.edu/monkmiddleware/public/analy tics/clusterclassification.html. [Accessed 17th June 2012]

[35] T. Kondo, J. Ueno, S. Takao, "Hybrid GMDH-type neural network using artificial intelligence and its application to medical image diagnosis of liver cancer", IEEE/SICE international, vol. 10, pp. 1101-1106, 2011.

[36] T. Matuki, T. Kondo, J. Ueno, "Three dimensional medical images of the lungs and brain recognized by artificial neural networks", SICE, Annual conference, vol. 10, pp. 1117-1121, 2007.

[37] M. F. Othman, M. A. M. Basri, "Probabilistic Neural Network for brain tumor classification", 2nd International Conference on Intelligent Systems, Modelling and Simulation, vol. 10, pp. 136$138,2011$.

[38] J. Anitha, C. K. S. Vijila, D. J. Hemanth, "An enchanced counter propagation neural network for abnormal retinal image classification", World Congress on Nature and Biology Inspired Computing, vol. 10, pp. 1-6, 2009 .

[39] F. Taher, N. Werghi, H. Al-Ahmad, R. Sammouda," Lung Cancer Detection by Using Artificial Neural Network and Fuzzy Clustering Methods", American Journal of Biomedical Engineering, vol. 2, pp. 136-142, 2012.

[40] S. D. Pathak, P. D. Grimm, V. Chalana, and Y. Kim. Pubic arch detection in transrectal ultrasound guided prostate cancer therapy. IEEE T. Med. Imag., vol. 17, pp. 762-771, 1998.

[41] K. T. Bae, M. L. Giger, C. Chen, and C. E. Kahn, "Automatic segmentation of liver structure in CT images", Med. Phys, vol. 20, pp.71-78, 1993

[42] L. Vincent and P. Soille, "Watersheds in digital spaces: an efficient algorithm based on immersion simulation", IEEE Trans. Patt. Anal. Mach. Intel., vol. 13, pp. 583-598, 1991. 183

[43] J. Sijbers, P. Scheunders, M. Verhoye, A. Van Der Linden, D. Van Dyck, et al. "Watershed-based segmentation of 3D MR data for volume quantization". Mag. Res. Imag., vol. 15, pp. 679-688, 1997.

[44] R. Loganathan, Y. S. Kumaraswamy, "An Improved Active Contour Medical Image Compression Technique with Lossless Region of Interest". 2011. Available at: http://ieeexplore.ieee.org/xpl/articleDetails.jsp?reload=true\&arnum ber $=6169098$.

[45] S.R. Gunn," Support Vector Machines for Classification and Regression”, Technical Report, pp. 17, 1998.

\section{BIOGRAPHY}

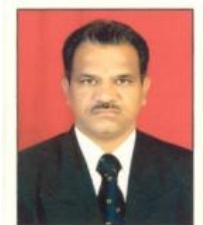

Shri N.D. Pergad, Associate Professor and Head of Electronics \& Telecommunication department at S.T.B. Engineering College, Tuljapur, DistrictOsmanabad, Maharashtra India. PhD (Registered) from Dr. Babasaheb Ambedkar Marathwada University, Aurangabad, Maharashtra. M.E from TKIE\&T Warananagar and B.E from M.S. Bidwe Engineering college, Latur. He has more than 15 publications to his credit and has been active in research and development. He has more than 24 years of teaching experience. He is the member of the IETE, ISTE. He is interested in the field of biomedical engineering, Digital Image Processing. Also recognised as P.G. Teacher in the Dr. B.A. Marathwada Univrsity, Aurangabad.

Professor in Instrumentation Engineering Dept., SGGS College of Engineering \& Technology, Nanded. (Autonomus \& Govt. Institute). 